\title{
INTERVALO HÍDRICO ÓTIMO E GRAU DE COMPACTAÇÃO DE UM LATOSSOLO VERMELHO APÓS 30 ANOS SOB PLANTIO DIRETO(1)
}

\author{
Edner Betioli Júnior ${ }^{(2)}$, Wagner Henrique Moreira ${ }^{(2)}$, Cássio Antônio \\ Tormena $^{(3)}$, Camila Jorge Bernabé Ferreira ${ }^{(4)}$, Álvaro Pires da Silva ${ }^{(5)}$ \\ \& Neyde Fabíola Balarezo Giarola ${ }^{(6)}$
}

\section{RESUMO}

A qualidade física do solo é um dos fatores determinantes da sustentabilidade agronômica, econômica e ambiental no sistema plantio direto (SPD). A compactação do solo tem sido apontada como um dos fatores de redução da qualidade física em solos sob SPD. Contudo, com a utilização do SPD, pode haver o incremento de matéria orgânica do solo e o desenvolvimento de um sistema poroso contínuo e estável, que atenuam os impactos negativos da compactação. O objetivo deste trabalho foi avaliar a qualidade física de um Latossolo Vermelho distroférrico sob SPD durante 30 anos, utilizando o intervalo hídrico ótimo (IHO) e o grau de compactação do solo (GC). Em uma área comercial com histórico de altas produtividades sob SPD, foram coletadas amostras de solo com estrutura preservada e deformada, para determinação da densidade do solo (Ds), do IHO e do GC. As amostras com estrutura preservada foram obtidas em três posições, relativas às linhas (L), entrelinhas (E) e posição intermediária entre as linhas e entrelinhas (PI) da cultura do milho. Foram determinadas as curvas de retenção de água e resistência do solo à penetração, bem como a Ds. A amostra de solo com estrutura deformada foi usada para obter a curva de compactação, utilizando o teste de Proctor. A Dmax foi obtida a partir da curva de compactação, e o GC foi determinado pela razão entre a Ds e a Dmax. Independentemente dos limites críticos de resistência à penetração (RP), verifica-se redução do IHO com o aumento da Ds. Os maiores valores do IHO foram verificados na posição de amostragem L, e a utilização de RP crítica maior que 2,0 MPa resultou em IHO condizente com a qualidade física desse solo sob SPD de longo tempo. A Dmax

(1) Recebido para publicação em 3 de maio de 2011 e aprovado em 27 de fevereiro de 2012.

(2) Mestrando do Programa de Pós Graduação em Agronomia, Universidade Estadual de Maringá - UEM. Avenida Colombo 5790. CEP 87020-900 Maringá (PR). E-mails: betioli.jr@gmail.com; wh.moreira@hotmail.com

(3) Professor associado do Departamento de Agronomia, UEM. Bolsista do CNPq. E-mail: catormena@uem.br

(4) Acadêmica do Curso de Agronomia - UEM. E-mail: kmilaphl@hotmail.com

(5) Professor Titular, Departamento de Solos e Nutrição de Plantas, Escola Superior de Agricultura "Luiz de Queiroz" - ESALQ/USP. Av. Pádua Dias 11 - Caixa Postal 09, CEP 13418-900 Piracicaba (SP). Bolsista do CNPq. E-mail: apisilva@esalq.usp.br

(6) Professora do Departamento de Ciência do Solo e Engenharia Agrícola, Universidade Estadual de Ponta Grossa - UEPG. Av. General Carlos Cavalcanti 4748, CEP 84030-900 Ponta Grossa (PR). E-mail: neydef@uepg.br 
foi de 1,52 $\mathrm{kg} \mathrm{dm}^{-3}$, e o GC variou de 64 a $87 \%$, sendo os maiores valores obtidos nas posições E e PI. Os valores de IHO e GC obtidos neste estudo indicam que a qualidade física desse solo não é limitante à produção das culturas após 30 anos de utilização do SPD.

Termos de indexação: qualidade física do solo, densidade do solo, estrutura do solo, resistência do solo à penetração.

\section{SUMMARY: LEAST LIMITING WATER RANGE AND DEGREE OF SOIL COMPACTION OF AN OXISOL AFTER 30 YEARS OF NO-} TILLAGE

The soil physical quality is one of the factors that determine the agronomic, economic and environmental sustainability in long-term no-tillage systems (NT). Soil compaction has been discussed as a factor that contributes to a reduction in physical quality under NT. However, the use of NT can increase soil organic matter and lead to a continuous and stable porous system that mitigates the negative impacts of compaction. The purpose of this study was to evaluate the physical quality of an Oxisol after 30 years of NT, using the least limiting water range (LLWR) and the degree of soil compaction (DC). The soil was sampled in a plantation with a history of high yields under NT. Soil samples with undisturbed and disturbed structure were collected to determine bulk density (BD), the LLWR and DC. The samples with undisturbed structure were taken from three sampling positions: from the rows $(R)$; from the middle in-between two rows $(M)$ and from an intermediate position (IP) between the $R$ and $M$ of corn plants. The water retention curve, soil resistance curve and $B D$ were determined. The disturbed soil sample was used to obtain the compaction curve using the Proctor test. The Dmax was obtained from the compaction curve and DC was determined by the ratio between the BD and Dmax. Regardless of the critical limits of resistance to penetration $(R P)$, there is a reduction of the LLWR with increasing $B D$. The highest values of LLWR were observed in the $R$ sampling position and the use of critical $R P>2.0 \mathrm{MPa}$ resulted in a LLWR consistent with the soil physical quality in long-term NT. The Dmax was $1.52 \mathrm{~kg} \mathrm{dm}^{-3}$ and DC ranged from 64 to $87 \%$, with the highest values obtained from the positions $M$ and IP. The values of LLWR and DC recorded in this study suggest that the physical quality of this soil after 30 years of NT is not limiting to crop production.

Index terms: soil physical quality, bulk density, soil structure, soil penetration resistance.

\section{INTRODUÇÃO}

O Sistema Plantio Direto (SPD) é um sistema de produção agrícola no qual é utilizado um conjunto de tecnologias caracterizadas pela ausência de revolvimento mecânico do solo, pela cobertura permanente do solo por resíduos culturais e pela utilização de rotação de culturas. Esses fundamentos são eficientes em promover o aumento do conteúdo de carbono orgânico e a melhoria da qualidade estrutural do solo. Em conjunto com as vantagens econômicas de redução do uso de maquinários, combustível e trabalho, os benefícios do SPD têm levado esse sistema de manejo a uma grande expansão, sendo atualmente praticado em aproximadamente 100 milhões de hectares por todo o mundo (Hobbs, 2007) e em cerca de 26 milhões de hectares no Brasil em 2006 (FEBRAPDP, 2011).
A utilização do SPD por longo prazo tem levantado questionamentos sobre os possíveis impactos negativos da compactação causada pelo tráfego de máquinas e ação de implementos, apesar de que o aumento de matéria orgânica pode contribuir para a atenuação da compactação do solo (BlancoCanqui et al., 2009). Há grandes divergências quanto aos efeitos da compactação do solo sobre a produtividade das culturas sob SPD, principalmente se consideradas as variáveis tempo de adoção do $\mathrm{SPD}$, tipo de solo, clima, manejo das culturas e, em particular, a condição estrutural do solo no momento da adoção do SPD (Ferreras et al., 2000). Em geral, solos sob SPD têm apresentado maior compactação nas camadas próximas à superfície, em relação aos solos sob manejo convencional (Veiga et al., 2007; Alvarez \& Steinbach, 2009). Contudo, as produtividades das culturas sob SPD são geralmente 
maiores ou equivalentes àquelas sob manejo convencional (Fabrizzi et al., 2005; Cardoso et al., 2007), sobretudo em regiões tropicais e subtropicais.

A melhoria da qualidade física do solo observada em áreas sob SPD de longo prazo tem sido atribuída à presença de agregados maiores e mais estáveis, em face do incremento de matéria orgânica, comparado com sistemas de preparo convencional (So et al., 2009; Jin et al., 2011). Outro fator positivo para a melhoria do ambiente físico do solo é a atividade da mesofauna e de raízes de culturas, que promovem uma rede de bioporos com maior continuidade e conectividade ao longo da matriz do solo, com reflexos no movimento de água (Strudley et al., 2008) e ar (Carter, 1992) no solo. Esses bioporos podem atuar como rotas alternativas para o crescimento das raízes (Ehlers et al., 1983; Bengough et al., 2011), apesar de uma matriz mais densa e com maior resistência mecânica.

Ao contrário do preparo convencional, a mobilização do solo no SPD restringe-se às linhas de semeadura, cuja intensidade depende do mecanismo utilizado para abertura do sulco. O uso de sulcadores pode reduzir a compactação superficial em SPD, devido à mobilização causada pelos mecanismos de corte do solo para abertura de sulcos pelas semeadoras (Veiga et al., 2007). Os efeitos combinados da abertura do sulco e do crescimento preferencial das raízes das plantas próximo às linhas resultam em um ambiente físico mais favorável nas linhas de plantio do que nas entrelinhas da cultura. Por sua vez, condições físicas mais favoráveis na linha podem induzir a formação de manchas de qualidade física, variáveis no espaço e tempo, em solos sob SPD.

Um indicador que tem sido utilizado para quantificar os impactos do uso e manejo na qualidade física do solo é o grau de compactação (GC) (Lipiec et al., 1991; Suzuki et al., 2007). O GC é resultado da razão entre a densidade do solo (Ds) obtida no campo e a densidade máxima do solo (Dmax) resultante de um teste de compactação, como o ensaio normal de Proctor, utilizado como referência (Dias Junior \& Miranda, 2000; Oliveira et al., 2010). Segundo Hakansson (1990) e Klein (2006), o GC elimina as influências da composição granulométrica, da mineralogia e da matéria orgânica do solo, facilitando a sua utilização no estudo e comparação de sistemas de uso e manejo dos solos.

O Intervalo Hídrico Ótimo (IHO) é um moderno indicador da qualidade física dos solos, por integrar numa só medida os efeitos do potencial da água no solo, da resistência mecânica à penetração das raízes e da porosidade do solo adequada à difusão de oxigênio às raízes (Letey, 1985; Silva et al., 1994;
Tormena et al., 2007). Entretanto, a definição dos níveis críticos de $\mathrm{RP}$ a serem utilizados nesse método depende de atributos como a condição estrutural do solo e o comportamento do sistema radicular das culturas. Taylor et al. (1966), Silva et al. (1994) e Tormena et al. (1998) utilizaram o valor crítico de $\mathrm{RP}=2,0 \mathrm{MPa}$, enquanto Ehlers et al. (1983) e Torres \& Saraiva (1999) sugerem valores maiores de RP em solos sob SPD, argumentando que as condições estruturais são menos restritivas ao crescimento radicular, provavelmente devido a um sistema poroso mais estável e com maior continuidade de poros.

No Brasil, poucos são os estudos que avaliam a qualidade física dos solos sob SPD após longo tempo de implantação (mais de 20 anos). Além disso, em SPD, os parâmetros físicos utilizados para avaliar a qualidade estrutural e física dos solos têm sido expressos pelo valor médio da área, sem levar em conta as mudanças físicas proporcionadas pelo posicionamento das linhas e entrelinhas das culturas. O objetivo deste trabalho foi avaliar a qualidade física de um Latossolo Vermelho distroférrico sob SPD após 30 anos de sua implantação, utilizando o Grau de Compactação e o Intervalo Hídrico Ótimo.

\section{MATERIAL E MÉTODOS}

A amostragem foi realizada numa área de lavouras comerciais localizada no município de Maringá - PR, situada a $23^{\circ} 30^{\prime} \mathrm{S}, 51^{\circ} 59^{\prime} \mathrm{W}$ e em altitude de $454 \mathrm{~m}$, apresentando relevo plano a suave ondulado, declividade média de $3 \%$, com médias anuais de temperatura e precipitação pluvial de $22{ }^{\circ} \mathrm{C}$ e $1.450 \mathrm{~mm}$, respectivamente. Nessa região o tipo climático dominante, segundo a classificação de Köppen, é o Cfa (subtropical úmido mesotérmico). $\mathrm{O}$ solo utilizado neste estudo foi classificado como Latossolo Vermelho distroférrico muito argiloso. O resultado da análise granulométrica na camada de 0-0,20 m indicou $750 \mathrm{~g} \mathrm{~kg}^{-1}$ de argila, $50 \mathrm{~g} \mathrm{~kg}^{-1} \mathrm{de}$ silte e $200 \mathrm{~g} \mathrm{~kg}^{-1}$ de areia.

A área, de 50 ha, é conduzida em sistema plantio direto (SPD) desde 1980 e utiliza rotação de culturas envolvendo milho, aveia, soja, trigo e milho de segunda safra. Durante os 30 anos de cultivo sob SPD foram feitas aplicações de calcário dolomítico, em superfície, com base nos resultados das análises de solo. Os fertilizantes foram aplicados por ocasião da semeadura com semeadora-adubadora equipada de discos de corte frontal e hastes com formato parabólico, ângulo de ataque de $20^{\circ}$, espessura da ponteira de $20 \mathrm{~mm}$ e profundidade de penetração entre 10 e $12 \mathrm{~cm}$. A cobertura com fertilizantes 
nitrogenados na cultura do milho foi feita com base nas recomendações para o material genético utilizado e produtividade esperada. O controle de pragas, doenças e plantas daninhas foi feito de acordo com as recomendações específicas para cada cultura. Na área amostrada, o tráfego de tratores, colhedoras e pulverizadores foi feito totalmente ao acaso, a fim de evitar zonas excessivamente compactadas.

A amostragem foi realizada em agosto de 2010, após a colheita do milho de segunda safra, cultivado com espaçamento de $0,90 \mathrm{~m}$. Foram coletadas, aleatoriamente, 12 amostras com estrutura preservada em cada uma das três posições de amostragem, relativas às linhas (L), entrelinhas (E) e posição intermediária entre as linhas e entrelinhas (PI) da cultura do milho, totalizando 36 amostras, as quais foram utilizadas para obtenção das curvas de retenção de água (CRA) e resistência do solo à penetração (CRS), da densidade do solo (Ds) e do intervalo hídrico ótimo (IHO). As amostras com estrutura preservada foram coletadas na camada de 0-0,10 m, em cilindros metálicos com dimensões aproximadas de $7,5 \mathrm{~cm}$ de diâmetro por $5,0 \mathrm{~cm}$ de altura, introduzidos lenta e continuamente por um amostrador elétrico automatizado (Figueiredo et al., 2010), para garantir a integridade estrutural do solo na amostra. Após a coleta, os cilindros com solo foram envolvidos com papel-alumínio e levados para o preparo no laboratório. O preparo das amostras consistiu na retirada do excesso de solo das extremidades de cada cilindro, de modo que o volume do solo fosse equivalente exatamente ao volume do cilindro. Em seguida, essas amostras foram saturadas durante $48 \mathrm{~h}$, por meio da elevação gradual de uma lâmina de água numa bandeja, até atingir cerca de dois terços da altura das amostras. Selecionaram-se ainda aleatoriamente 20 pontos, nos quais foram coletados aproximadamente $3,5 \mathrm{~kg}$ de solo com estrutura deformada, na camada de 0-0,10 m, totalizando $70 \mathrm{~kg}$ de solo, que foram utilizados para obtenção da curva de compactação e da densidade máxima do solo (Dmax) ou de referência.

Para a determinação da Dmax foi utilizado o ensaio de Proctor normal em três repetições. As amostras deformadas de solo coletadas na camada de $0-0,10 \mathrm{~m}$ foram secas ao ar e, posteriormente, passadas em peneira com abertura de malha de $4 \mathrm{~mm}$. O solo seco ao ar apresentava teor residual de água de aproximadamente $0,06 \mathrm{~kg} \mathrm{~kg}^{-1}$. As amostras foram compactadas em seis teores crescentes de água, permitindo a obtenção de diferentes densidades e caracterizando a curva de compactação do solo. A compactação do solo foi feita num cilindro metálico de $1.000 \mathrm{~cm}^{3}$, em três camadas consecutivas, cada uma recebendo 25 golpes de um soquete de $2,5 \mathrm{~kg}$ caindo de uma altura de $0,3 \mathrm{~m}$, correspondendo a uma energia de $560 \mathrm{kPa}$, conforme Nogueira (1998). Após a compactação, foi retirada uma porção de solo do corpo de prova, para determinação do teor de água.

A determinação da CRA nas amostras indeformadas foi feita de acordo com o procedimento descrito por Tormena et al. (1998). As amostras foram divididas em nove grupos de quatro amostras, que foram submetidos aos potenciais de $-2,-4,-6,-8,-10 \mathrm{kPa}$ numa mesa de tensão, conforme Ball \& Hunter (1988), e de -30, -100, - 400 e $-1.500 \mathrm{kPa}$, utilizando pressões aplicadas em câmaras com placas porosas, conforme Klute (1986). O teor de água no potencial de $-1,5 \mathrm{MPa}$, equivalente ao ponto de murcha permanente, foi posteriormente estimado com uso do equipamento Dewpoint Potentiameter (WP4-T), conforme Decagon Devices (2011), utilizando as quatro amostras anteriormente submetidas ao potencial de -1,5 MPa nas placas porosas. Esse procedimento foi necessário para melhorar a estimativa do teor de água no potencial de -1,5 MPa, em face dos erros experimentais associados com o uso de placas, conforme Bittelli \& Flury (2009).

Após atingir o equilíbrio hidráulico em cada potencial aplicado nas placas porosas, as amostras foram pesadas e, em seguida, foi determinada a resistência do solo à penetração $(\mathrm{RP})$ utilizando o equipamento e os procedimentos descritos em Tormena et al. (1999). Posteriormente, as amostras foram secas em estufa a $\pm 105^{\circ} \mathrm{C}$, por $24 \mathrm{~h}$, para determinação da massa de sólidos e da massa de água no solo. A Ds foi determinada pela razão entre a massa de sólidos e o volume do cilindro. O teor de água em base de volume $(\theta)$ foi obtido pelo produto do teor de água em base de massa e a Ds.

A descrição matemática da CRS e da CRA faz-se necessária para a determinação do IHO. A CRA, expressa pela relação entre o $\theta$ e o potencial da água no solo $(\Psi)$, foi descrita matematicamente por meio de uma função não linear, incorporando a variação da Ds entre as amostras, segundo Leão et al. (2005), pela equação 1 :

$$
\theta=(a+b D s) \Psi^{c}
$$

em que $\theta$ : teor de água do solo $\left(\mathrm{m}^{3} \mathrm{~m}^{-3}\right)$; Ds: densidade do solo $\left(\mathrm{kg} \mathrm{dm}^{-3}\right) ; \Psi$ : potencial da água no solo $(\mathrm{hPa}) ; a, b$ e $c$ : parâmetros obtidos no ajuste do modelo aos dados de $\theta(\Psi)$.

A relação funcional entre a RP, $\theta$ e Ds estabelece a CRS, a qual tem sido adequadamente modelada utilizando uma função não linear proposta por Busscher \& Sojka (1987) e Busscher (1990), descrita na equação 2 : 


$$
R P=d \theta^{e} D s^{f}
$$

em que a RP: resistência do solo à penetração $(\mathrm{MPa})$; $\theta$ : teor de água do solo $\left(\mathrm{m}^{3} \mathrm{~m}^{-3}\right)$; Ds: densidade do solo $\left(\mathrm{kg} \mathrm{dm}^{-3}\right) ; d$, e e $f$ : parâmetros obtidos no ajuste do modelo aos dados.

O IHO foi determinado adotando os procedimentos descritos em Silva et al. (1994) e Tormena et al. (1999). Os valores de $\theta$ associados com o potencial mátrico, a RP e a porosidade de aeração foram, respectivamente: a umidade na capacidade de campo ( $\theta c c)$ ou teor de água estimado no potencial de $-10 \mathrm{kPa}$ (Reichardt, 1988); a umidade no ponto de murcha permanente $(\theta \mathrm{pmp})$ ou teor de água no potencial de -1.500 kPa (Savage et al., 1996); e o teor de água do solo em que a porosidade de aeração ( $\theta$ pa) é de $0,10 \mathrm{~m}^{3} \mathrm{~m}^{-3}$ (Grable \& Siemer, 1968). Para a RP foram adotados os valores críticos de 2,0 $\mathrm{MPa}$. Adicionalmente, foram utilizados os valores críticos de $\mathrm{RP}=3,5 \mathrm{MPa}$, conforme Torres \& Saraiva (1999), e $\mathrm{RP}=4,6 \mathrm{MPa}$, com base nos resultados de Ehlers et al. (1983). Os valores de $\theta$ cc e $\theta$ pa foram obtidos nos potenciais de -10 e $-1.500 \mathrm{kPa}$, utilizando a $\mathrm{CRA}$, e os valores do $\theta$ em que a $\mathrm{RP}$ atinge os valores críticos ( $\theta \mathrm{rp}$ ) foram obtidos por meio da CRS. O valor de $\theta \mathrm{pa}$, o teor de água no solo em que a porosidade de aeração é de $0,10 \mathrm{~m}^{3} \mathrm{~m}^{-3}$, foi obtido por [(1-Ds/ Dp)-0,1]. Considerou-se o valor médio de densidade de partículas do solo (Dp) de $2,85 \mathrm{~kg} \mathrm{dm}^{-3}$, obtido conforme Embrapa (1997).

Os resultados da curva de compactação, ou seja, Ds em função do $\theta$, foram ajustados a uma equação polinomial de segundo grau, minimizando a soma dos quadrados dos desvios. O teor de água ou umidade crítica para compactação (Uc) foi obtido por meio da primeira derivada da equação polinomial de segundo grau ajustada aos dados. A Dmax foi obtida pela utilização do valor de Uc na equação originalmente obtida. O grau de compactação do solo (GC) foi estimado pela razão entre a Ds e a Dmax ou de referência, conforme Hakansson (1990).

Os ajustes dos dados à CRA e CRS foram feitos empregando o software SAS Institute (1999). As comparações dos atributos físicos entre as posições de amostragem foram feitas utilizando o intervalo de confiança da média $(\mathrm{p}<0,05)$, conforme Payton et al. (2000). Quando não há sobreposição dos limites superior e inferior do intervalo de confiança, considera-se que há diferença significativa.

\section{RESULTADOS E DISCUSSÃO}

Os valores médios dos parâmetros físicos, determinados nas amostras com estrutura preservada obtidas nas diferentes posições de amostragem, são apresentados no quadro 1.

A resistência do solo à penetração (RP) é caracterizada pela elevada variabilidade dos dados (CV> 59\%), já verificada em outros trabalhos (Imhoff et al., 2000; Blainski et al., 2009). O máximo valor de $\mathrm{RP}$ foi de 4,43 MPa, bem abaixo dos valores obtidos por Tormena et al. (1999) e Tormena et al. (2007) em Latossolos Vermelhos de similar classe textural. Ressalta-se que a área é bastante homogênea em

Quadro 1. Valores médios dos atributos físicos das amostras com estrutura preservada obtidas nas diferentes posições de amostragem

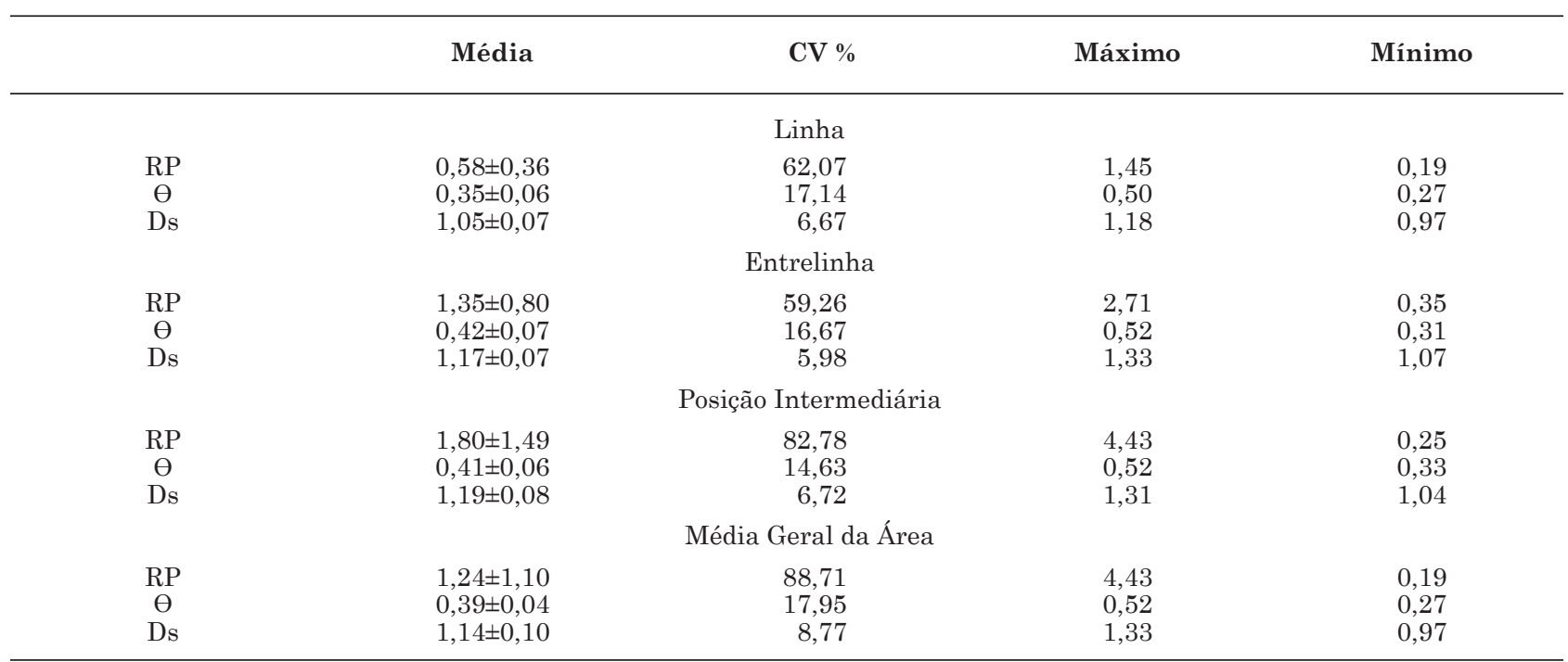

CV: coeficiente de variação (\%); RP: resistência do solo à penetração (MPa); $\theta$ : umidade do solo $\left(\mathrm{m}^{3} \mathrm{~m}^{-3}\right)$; Ds: densidade do solo $\left(\mathrm{kg}\right.$ dm $\left.{ }^{-3}\right)$. 
termos de textura e que a amostragem foi realizada utilizando a introdução lenta e contínua do cilindro, por meio de um amostrador elétrico automatizado (Figueiredo, 2010), o qual garante a manutenção da integridade estrutural do solo na amostra.

A curva de compactação do solo da área estudada, utilizando o método de Proctor, é apresentada na figura 1, na qual a curva refere-se aos valores de Ds e teor de água (U) das três repetições; uma vez que as curvas foram similares, o ajuste da função polinomial de segundo grau aos dados foi feito utilizando 18 pontos de Ds e U, para obtenção de uma curva média.

O ajuste dos dados ao modelo quadrático apresentou todos os parâmetros significativos $(\mathrm{p}<0,0001)$ e resultou num coeficiente de determinação de 0,93, de acordo com Beutler et al. (2005), Vieira \& Klein (2007) e Oliveira et al. (2010). A qualidade do ajuste do modelo aos dados pode ser considerada adequada principalmente levando-se em conta a utilização de 18 pontos de Ds e U. Os valores de Uc e Dmax foram de $0,29 \mathrm{~kg} \mathrm{~kg}^{-1}$ e $1,52 \mathrm{~kg} \mathrm{dm}^{-3}$, respectivamente. Num Latossolo Vermelho com $520 \mathrm{~g} \mathrm{~kg}^{-1}$ de argila e 3,7 \% de matéria orgânica, Beutler et al. (2005) obtiveram um valor de Dmax $=1,54 \mathrm{~kg} \mathrm{dm}^{-3}$. Figueiredo et al. (2000)

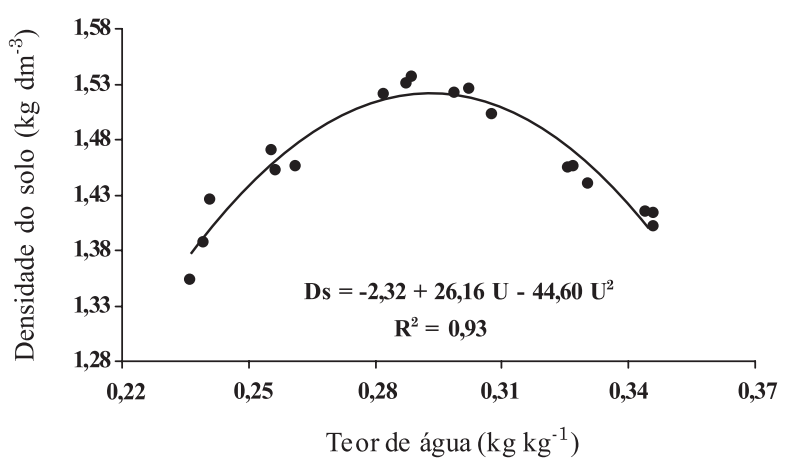

Figura 1. Densidade do solo (Ds) em função do teor de água (U) determinada por meio do teste de Proctor. verificaram $\mathrm{Uc}=0,27 \mathrm{~kg} \mathrm{~kg}^{-1} \mathrm{e} D \max =1,51 \mathrm{~kg} \mathrm{dm}^{-3}$ para um Latossolo Vermelho, sob plantio direto, com $650 \mathrm{~g} \mathrm{~kg}^{-1}$ de argila e 3,0 \% de matéria orgânica. A similaridade dos valores de Dmax obtida pelo teste de Proctor nos diferentes estudos com solos similares indicam, conforme Klein (2006), a adequação desse método com boa repetibilidade para obtenção de valores referenciais de Dmax, para fins de manejo e monitoramento da qualidade física dos solos.

$\mathrm{O}$ teor de água no solo $\left(\mathrm{Uc}=0,29 \mathrm{~kg} \mathrm{~kg}^{-1}\right)$ que resultou na máxima densidade está ligeiramente abaixo da capacidade de campo para esse solo $\left(0,36 \mathrm{~kg} \mathrm{~kg}^{-1}\right)$, condição em que o tráfego de máquinas deveria ser evitado. A capacidade de campo foi obtida da figura 2, por meio da divisão do teor de água na capacidade de campo pela Ds. De acordo com Koolen (1994), o incremento do teor de água aumenta a suscetibilidade à compactação, uma vez que reduz a resistência interna do solo e facilita o processo de deformação. Por outro lado, a diminuição no teor de água implica redução do potencial da água no solo, estabelecendo maior coesão e atrito entre as partículas e, portanto, menor suscetibilidade do solo à compressão (Weirich Neto et al., 2002). A manutenção da qualidade física desse solo em sistema de plantio direto sob longo prazo exige cuidados em relação à umidade do solo em que ocorre o tráfego de máquinas pesadas, sobretudo na colheita. Especificamente nesse solo, conforme pode ser verificado na figura 1 , pequenas variações do teor de água implicam significativos incrementos da densidade até o teor de água limite para a máxima compactação do solo.

O grau de compactação (GC) tem sido utilizado para contornar a problemática da comparação de resultados de densidade entre solos que apresentam diferentes características mineralógicas, de textura e de matéria orgânica, conforme reportado por Hakansson (1990) e Klein (2006). Os valores médios de GC do solo estudado são apresentados no quadro 2.

A posição de amostragem Linha (L) apresentou GC estatisticamente inferior às demais posições (não

Quadro 2. Valores médios, máximos e mínimos para o grau de compactação do solo (\%) (GC) nas diferentes posições de amostragem

\begin{tabular}{lccccc}
\hline Posição & Média & Máximo & Mínimo & CV (\%) & IC \\
\hline Linha & $69,05 \pm 4,46$ & 77,82 & 63,78 & 6,46 & 2,53 \\
Entrelinha & $77,10 \pm 4,52$ & 87,43 & 70,35 & 5,86 & 2,56 \\
Intermediário & $78,50 \pm 5,25$ & 85,92 & 68,73 & 6,69 & 2,97 \\
Média Geral & $74,89 \pm 6,26$ & 87,43 & 63,78 & 8,36 & - \\
\hline
\end{tabular}

CV: coeficiente de variação; IC: intervalo de confiança $(p<0,05)$. 
sobreposição dos intervalos de confiança), refletindo os efeitos do revolvimento superficial causado pela haste sulcadora durante a operação de semeadura da cultura do milho e, posteriormente, ampliado pelo crescimento do sistema radicular da cultura. Verifica-se que os valores médios e máximos de GC da área estão no intervalo de valores estipulados na literatura como ideais para o máximo rendimento das culturas, estimados entre 77 e $94 \%$.

Diversos autores sugerem valores de GC ótimos para o desenvolvimento das culturas ou, ainda, críticos a elas. Em estudo com solos arenosos, Carter (1990) indica que a faixa de GC de 77 a $84 \%$ proporcionou produção de cereais superior a $95 \%$ da máxima. Esse autor também salienta que, na faixa de GC entre 84 e $89 \%$, o volume de macroporos variou de 13,5 a 10,0 \%, indicando não haver limitações físicas às plantas por aeração. Hakansson (1990) relata o máximo rendimento de cevada para um GC próximo a 87 \%. Já Lipiec et al. (1991) indicam valores ótimos de GC de 88 e $91 \%$ para o crescimento de plantas e rendimento de grãos de cevada, respectivamente. No Brasil, Suzuki et al. (2007) indicam que um GC de 86 \% (obtido utilizando a Dmax de referência em uma pressão de $1.600 \mathrm{kPa}$ ) pode ser considerado ótimo para a cultura da soja, enquanto Beutler et al. (2005) verificaram, por meio do teste de Proctor, um GC ótimo de 80 \%. Com base nos valores de GC obtidos na literatura, os resultados médios de GC da área sugerem que esta se encontra em boa condição física, considerados os resultados de GC associados com condições físicas altamente satisfatórias às plantas.

O GC na posição L variou de 63 a $77 \%$, sendo $66 \%$ dos dados inferiores ao valor mínimo encontrado nas demais posições (69 \%). Para a posição intermediária (PI) a variação foi de 69 a $86 \%$, e na posição entrelinha (E), de 70 a $87 \%$. A PI apresentou $50 \%$ dos dados com GC acima de $80 \%$, enquanto em $\mathrm{E}$ esse valor foi de $15 \%$. O máximo valor de GC foi obtido nas posições E e PI, resultante da compactação do solo pelas operações com máquinas na área, ao passo que o menor valor em L é derivado do revolvimento do solo por ocasião da semeadura, bem como pela atuação do sistema radicular da cultura do milho. No entanto, os valores mínimos em $\mathrm{E}$ e PI estão próximos do valor médio em L, sugerindo que na E e PI ocorrem baixos valores de $\mathrm{GC}$ associados à variabilidade espacial e temporal da Ds em função da provável presença de linhas de culturas anteriores.

As funções descritivas das curvas de retenção de água (CRA) e de resistência do solo à penetração (CRS) são mostradas no quadro 3. O ajuste das funções aos dados mostrou-se estatisticamente significativo, uma vez que os intervalos de confiança dos parâmetros das funções não incluíram o valor zero, conforme Grantz \& Slinker (1990). A CRA foi significativa e positivamente influenciada pela $\mathrm{Ds}$, a exemplo dos trabalhos de Petean et al. (2010) e Klein \& Camara (2007), em solos de mesma classe e teores similares de argila. A influência positiva da Ds e negativa de $\theta$ na CRS é amplamente reportada na literatura (Imhoff et al., 2001; Leão et al., 2004; Figueiredo et al., 2011). O efeito positivo da Ds deve-se ao maior atrito entre as partículas e à coesão, enquanto variações de $\theta$ promovem efeitos associados à coesão entre partículas devido à variação de potencial e ao efeito lubrificante da água (Vepraskas, 1984).

As funções de CRA e CRS descritas no quadro 3 foram utilizadas para estimar os teores de água $(\theta)$ na capacidade de campo $(\theta \mathrm{cc})$, no ponto de murcha permanente $(\theta \mathrm{pmp})$ e na resistência crítica do solo à penetração ( $\theta \mathrm{rp})$, os quais, juntamente com $\theta$, em que a porosidade com ar é de $10 \%(\theta \mathrm{pa})$, são apresentados na figura 2 . Diferentes valores críticos de RP têm sido reportados na literatura, porém o valor de $\mathrm{RP}=2,0 \mathrm{MPa}$ é o mais frequentemente utilizado como crítico para o crescimento das plantas (Taylor et al., 1966; Silva et al., 1994; Tormena et al., 1998; Bengough et al., 2011). Outros pesquisadores têm sugerido que em solos sob SPD o limite crítico de RP deve ser ampliado além de 2,0 $\mathrm{MPa}$, devido à diferente organização e funcionalidade do sistema poroso dos solos intensamente revolvidos (Torres \& Saraiva, 1999; Hakansson \& Lipiec, 2000).

A figura 2a mostra o IHO para a área estudada, utilizando a $\mathrm{RP}=2,0 \mathrm{MPa}$ como crítica. Torres \&

Quadro 3. Equações da curva de retenção de água no solo e resistência do solo à penetração e respectivas estatísticas

\begin{tabular}{cccc}
\hline Função & Equação & F & P \\
\hline CRA & $\theta=(0,1721+0,4171 D s) \Psi(-0,098)$ & 3645,25 & $<0,0001$ \\
CRS & $R P=0,00147 \theta^{-4,9838} D s^{12,7773}$ & $131,67<0,0001$ \\
\hline
\end{tabular}

$R P$ : resistência à penetração $(\mathrm{MPa}) ; D s$ : densidade do solo $\left(\mathrm{kg} \mathrm{dm}^{-3}\right)$; e $\theta$ : teor de água $\left(\mathrm{m}^{3} \mathrm{~m}^{-3}\right)$. 
Saraiva (1999) sugerem que, para a cultura da soja em Latossolos Vermelhos argilosos, valores de RP na faixa de 3,5 a $6,5 \mathrm{MPa}$ são os mais indicados para considerar que o solo está com possíveis problemas de impedimento mecânico às raízes. Assim, na figura $2 \mathrm{~b}$ o IHO foi estimado utilizando a $\mathrm{RP}=3,5 \mathrm{MPa}$, introduzido como limite máximo de RP crítico. Nesse contexto, Ehlers et al. (1983), estudando áreas sob plantio direto e preparo convencional num solo de clima temperado, sugerem que para solos sob plantio direto o valor crítico de $\mathrm{RP}$ varia de 4,6 a $5,1 \mathrm{MPa}$, sob o argumento de que o crescimento radicular nesses solos pode ser beneficiado pela presença de macroporos contínuos decorrentes da atividade biológica (mesofauna e raízes). Na figura $2 \mathrm{c}$ é apresentado o IHO utilizando um valor de RP crítico de 4,6 $\mathrm{MPa}$, conforme indicado por Ehlers et al. (1983).

$\mathrm{Na}$ figura 2, também verifica-se que a água disponível entre $\theta c c$ e $\theta \mathrm{pmp}$ aumenta com a Ds, o que é indicado pelo sinal positivo da Ds na função de retenção ajustada aos dados (Quadro 3). Por outro lado, o $\theta$ em que o volume de poros com ar é de $10 \%$ reduz com o aumento da Ds. No entanto, constata-se que, apenas em poucos valores de Ds, $\theta$ par assume valores menores do que $\theta c c$, sugerindo que a aeração não é a condição física limitante para o crescimento das plantas. Resultados similares foram obtidos por Tormena et al. $(1999,2007)$ e Blainski et al. (2009). Similarmente à maioria dos trabalhos que medem o IHO em solos brasileiros, verifica-se que a RP é a principal variável associada com a redução da qualidade física, o que é constatado na figura 2 , na qual o IHO é reduzido até zero com o aumento da Ds (Figura 2a,b). A constatação da RP como principal fator físico limitante às plantas também é respaldada por Bengough et al. (2011), visto que a variação de $R P$ é dependente da variação de $\theta$ e influenciada pelo aumento de Ds ou GC.

Independentemente dos limites críticos de RP adotados, verifica-se redução do IHO com o aumento da Ds, caracterizando perda da qualidade física com o aumento da compactação do solo. Utilizando o valor de $\mathrm{RP}=2,0 \mathrm{MPa}$ como crítico, as condições físicas do solo apresentam-se mais restritivas às plantas do que com a $\mathrm{RP}=3,5$ e 4,6 $\mathrm{MPa}$, porém, em todos os casos, a RP é dominante na redução do IHO. A ampliação do limite de RP aumentou o valor de Ds em que a RP substitui PMP como limite inferior do IHO, bem como ampliou o valor de Ds crítica para valores acima de $1,32 \mathrm{~kg} \mathrm{dm}^{-3}$, comparado com a Ds crítica $=1,29 \mathrm{~kg} \mathrm{dm}^{-3}$, utilizando $\mathrm{RP}=2,0 \mathrm{MPa}$. A ampliação do IHO utilizando uma RP crítica de 3,5 a $4,6 \mathrm{MPa}$ é compatível com a boa qualidade física desse solo, tendo em vista o histórico de altas produtividades da área ao longo desses últimos anos de SPD. Esses resultados sugerem que o monitoramento da compactação e da qualidade física do solo com uso de penetrômetros exige que o limite crítico de RP seja ampliado, assim como são necessários estudos para definição dos valores críticos de resistência em solos sob SPD de longo prazo. Para RP crítica acima desses valores, a aeração e a retenção de água constituem-se nos fatores mais restritivos para o IHO do que a RP.

A variação do IHO com o GC do solo é mostrada na figura 3. Verifica-se ampla faixa de IHO e GC em todas as posições de amostragem; os menores valores de GC e os maiores valores de IHO predominam na
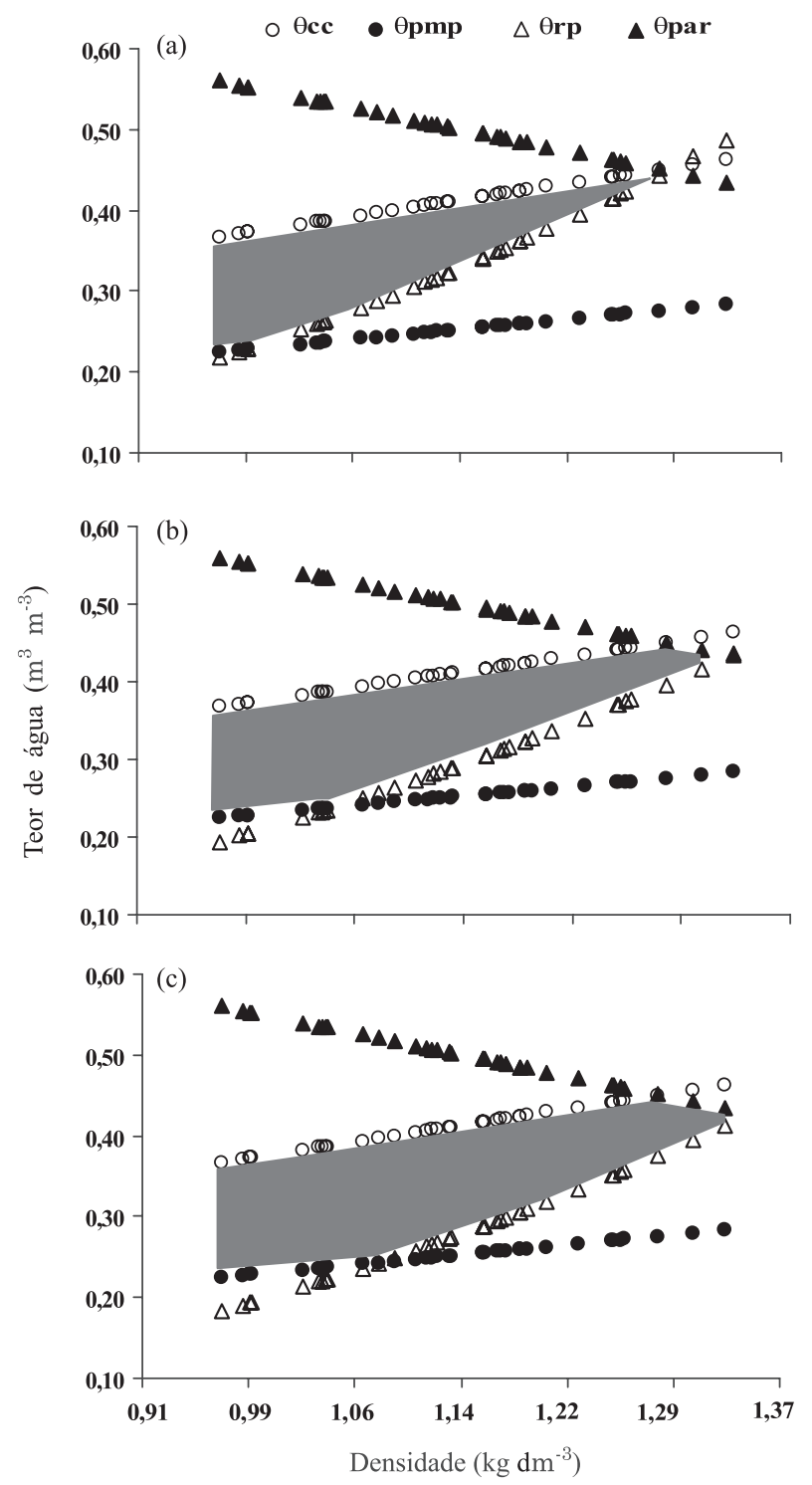

Figura 2. Variação dos teores de água do solo na capacidade de campo $(\theta \mathrm{cc})$, ponto de murcha permanente $(\theta \mathrm{pmp})$, porosidade com ar de $10 \%$ ( $\theta$ par) e resistência do solo à penetração $(\theta \mathrm{rp})$ de 2,0 MPa (a), 3,5 MPa (b) e 4,6 MPa (c), em função da densidade do solo. A área em cor cinza corresponde ao intervalo hídrico ótimo do solo. 
posição L, associados com o revolvimento superficial do solo ocorrido durante a semeadura e com o efeito do sistema radicular da cultura do milho. Esses resultados estão em acordo com os de Correchel et al. (1999), que também constataram menor Ds na linha da cultura, comparado à entrelinha. O aumento do GC implica redução do IHO, com os menores valores em PI e E, resultante da compactação pelo tráfego de máquinas na área. Os resultados sugerem que há variação espacial da qualidade física associada à mudança temporal das linhas das culturas em SPD. As condições físicas mais favoráveis nas linhas das culturas em solos sob SPD devem-se à ação de revolvimento superficial do solo pelos sulcadores utilizados para inserir o fertilizante ao lado e abaixo das sementes.

O IHO para a RP de 2,0 MPa correlaciona-se positivamente até o GC de $65 \%$, enquanto o IHO para as RPs de 3,5 e 4,6 MPa correlacionam-se positivamente até valores de GC de 69 e $71 \%$, respectivamente. Nesses valores de GC são verificados os máximos valores do IHO. O GC crítico, ou o valor de GC no qual o IHO $=0$, foi de $85 \%$ para $\mathrm{RP}=2,0 \mathrm{MPa}$ e superior a $87 \%$ para as demais RPs. O GC de $85 \%$, tido como limitante para $\mathrm{RP}=2,0 \mathrm{MPa}$, não condiz com os valores de GC reportados como ideais para a produtividade ótima das culturas, que variam entre 86 e $91 \%$ (Hakansson, 1990; Lipiec et al., 1991; Suzuki et al., 2007). Essa constatação é contraditória, pois nesse caso os valores de GC ótimos para a produtividade das culturas correspondem a valores que já superaram o limite no qual o IHO é nulo. Isso sugere que a adoção de $\mathrm{RP}=2,0 \mathrm{MPa}$ como valor crítico pode superestimar a condição restritiva ao desenvolvimento do sistema radicular das plantas em SPD no longo prazo, desde que o sistema de manejo dessas áreas estabeleça um sistema poroso estável e com bioporos abundantes e contínuos, que permita aumentar os limites críticos de RP. Neste estudo, a utilização de RP maior que 2,0 $\mathrm{MPa}$ é

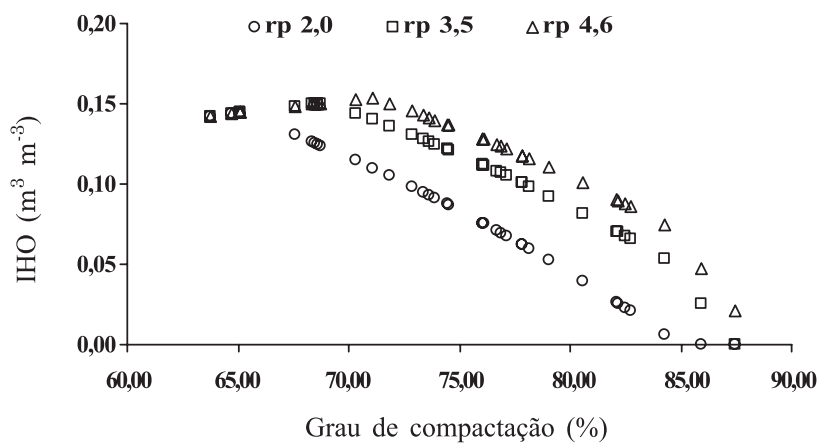

Figura 3. Variação do intervalo hídrico ótimo em função do grau de compactação do solo para os valores de resistência à penetração de 2,0, 3,5 e 4,6 MPa. mais compatível com os resultados de GC ótimos para produtividade das culturas, por evidenciar uma condição de menor restrição mecânica para o aproveitamento da água disponível no solo.

A relação entre o GC e o rendimento das culturas ocorre de forma indireta, uma vez que o GC altera propriedades físicas diretamente ligadas ao crescimento das plantas, a exemplo do que foi constatado por Suzuki et al. (2007). Dessa forma, valores ótimos de GC para as culturas dependem da composição granulométrica e da estrutura do solo (Lipiec et al., 1991; Suzuki et al., 2007), e a magnitude desses valores de GC pode variar no tempo em função das condições climáticas, especialmente da disponibilidade hídrica e das culturas envolvidas (Carter, 1990; Hakansson, 1990; Lipiec et al., 1991). Por sua vez, o IHO tem a vantagem de ser um parâmetro físico definido pelos teores de água nos limites críticos de potencial, aeração e resistência do solo à penetração, os quais têm relação com processos físiológicos que definem o crescimento e rendimento das culturas (Dodd et al., 1996; Masle, 1998). Utilizando o valor de $\mathrm{GC}=80 \%$, definido por Beutler et al. (2005) como ótimo para a cultura da soja, os resultados sugerem que o solo estudado apresenta condições físicas altamente favoráveis após longo tempo sob SPD. Os resultados obtidos por Suzuki et al. (2007) num solo similar ao deste estudo indicam que o GC ótimo para a cultura da soja em SPD é de $86 \%$, o que pode estar relacionado ao fato de que a densidade de referência foi obtida utilizando uma energia equivalente à carga de $1.600 \mathrm{kPa}$. Apesar da ampla faixa de valores de GC obtidos no presente estudo (64 a 87 \%), não há indicações de limitações físicas às plantas devido à aeração e resistência, e os valores de GC menores que os referenciados na literatura não indicam, a priori, comprometimento da retenção de água no solo.

É necessário ressaltar que a magnitude do IHO é dependente dos níveis críticos das propriedades envolvidas, em especial da resistência do solo à penetração, a qual, para a maioria dos estudos, é considerada a principal propriedade física que limita o crescimento das plantas e, por consequência, o IHO (Silva et al., 2009; Bengough et al., 2011). Considerando os valores de GC obtidos neste estudo e assumindo os valores ótimos de GC para as culturas indicados na literatura, os resultados mostrados nas figuras 2 e 3 sugerem que, nesse solo sob SPD por longo prazo, valores de IHO obtidos com $\mathrm{RP}>2,0 \mathrm{MPa}$ são condizentes com uma qualidade física do solo adequada às altas produtividades das culturas de milho, soja e trigo na área estudada. Por exemplo, o maior valor de GC encontrado em $\mathrm{L}(77 \%)$ corresponde ao IHO de $0,07 \mathrm{~m}^{3} \mathrm{~m}^{-3}$, que é equivalente à metade do IHO máximo, obtido com 
$\mathrm{RP}=2,0 \mathrm{MPa}$, sugerindo que o desenvolvimento das plantas não seria restringido nessa condição e posição de amostragem.

A manutenção do sistema de rotação de culturas, de adequada cobertura e a ausência de revolvimento do solo nesse período permitiram o estabelecimento e manutenção de boas condições físicas no solo via sistema poroso estável e contínuo, o que possibilita ampliar os limites de RP nesse solo. Adicionalmente, o controle do teor de água por meio da cobertura do solo possibilita o aproveitamento da boa condição estrutural via manutenção da umidade dentro dos limites do IHO e constitui uma estratégia importante para o manejo físico dos solos sob SPD. Em plantio direto, a bioporosidade resultante da reduzida movimentação mecânica do solo pode oferecer caminhos alternativos para o crescimento das raízes, compensando a maior resistência da matriz do solo. Por isso, o limite de resistência crítica de 2,0 MPa pode ser ampliado no cálculo do IHO, o que, em termos práticos, significa ampliar o secamento do solo sem que haja restrições mecânicas às plantas, a exemplo de Tormena et al. (2007), que estabeleceram diferentes níveis de RP para distinguir diferentes sistemas de manejo em plantio direto, devido à maior incidência de bioporos no solo com o uso de rotação de culturas.

\section{CONCLUSÕES}

1. Independentemente dos limites críticos de $\mathrm{RP}$ adotados, verifica-se redução do IHO com o aumento da Ds. Os maiores valores do IHO foram verificados na posição de amostragem relativa à linha da cultura, e a utilização de RP crítica maior que 2,0 $\mathrm{MPa}$ resultou em IHO condizente com a qualidade física desse solo sob SPD de longo tempo.

2. A densidade do solo máxima foi de $1,52 \mathrm{~kg} \mathrm{dm}^{-3}$, e o grau de compactação (GC) variou de 64 a $87 \%$; os maiores valores foram obtidos nas posições relativas à entrelinha das culturas e na posição intermediária entre a linha e a entrelinha da cultura.

3. Os valores de IHO e GC obtidos neste estudo indicam que a qualidade física desse solo não é limitante à produção das culturas após 30 anos de uso do SPD, nas condições do solo e manejo estudados.

\section{LITERATURA CITADA}

ALVAREZ, R. \& STEINBACH, H.S. A review of the effects of tillage systems on some soil physical properties, water content, nitrate availability and crops yield in the Argentine Pampas. Soil Tillage Res., 104:1-15, 2009.
BALL, B.C. \& HUNTER, R. The determination of water release characteristics of soil cores at low suctions. Geoderma, 43:195-212, 1988.

BEnGOUGH, A.G.; McCKEnZIE, B.M.; HALLETT, P.D. \& VALENTINE, T.A. Root elongation, water stress, and mechanical impedance: A review of limiting stresses and beneficial root tip traits. J. Exper. Bot., 62:59-68, 2011.

BEUTLER, A.N.; CENTURION, J.F. \& SIlVA, A.P. Soil resistance to penetration and least limiting water range for soybean yield in a haplustox from Brazil. Braz. Arch. Biol. Technol., 48:863-871, 2005.

BITTELLI, M. \& FLURY, M. Errors in water retention curves determined with pressure plates. Soil Sci. Soc. Am. J., 73:1453-1460, 2009.

BLAINSKI, E.; GONÇALVES, A.C.A.; TORMENA, C.A.; FOLEGATTI, M.V. \& GUIMARÃES, R.M.L. Intervalo hídrico ótimo num Nitossolo Vermelho distroférrico irrigado. R. Bras. Ci. Solo, 33:273-281, 2009.

BLANCO-CANQUI, H.; STONE, L.R.; SCHLEGEL, A.J.; LYON, D.J.; VIGIL, M.F.; MIKHA, M.M.; STAHLMAN, P.W. \& RICE, C. No-till induced increase in organic carbon reduces maximum bulk density of soils. Soil Sci. Soc. Am. J., 73:1871-1879, 2009.

BUSSCHER, W.J. \& SOJKA, R.E. Enhancement of subsoiling effect on soil strength by conservation tillage. Trans. ASAE, 30:888-892, 1987.

BUSSCHER, W.J. Adjustment of flat-tipped penetrometer resistance data to common water content. Trans. Am. Soc. Agric. Eng., 3:519-524, 1990.

CARDOSO, E.G.; ZOTARELLI, L.; PICCININ, J.L..; TORRES, E.; SARAIVA, O.F. \& GUIMARÃES, M.F. Soybean root system in function of soil compaction under no-tillage system. Pesq. Agropec. Bras., 41:493-501, 2007.

CARTER, M.R. Characterizing of soil physical condition in reduced tillage systems for winter wheat on a fine sandy loam using small cores. Can. J. Soil Sci., 72:395-402, 1992.

CARTER, M.R. Relative measures of soil bulk density to characterize compaction in tillage studies on fine sandy loams. Can. J. Soil Sci., 70:425-433, 1990.

CORRECHEL, V.; SILVA, A.P. \& TORMENA, C.A. Influência da posição relativa à linha de cultivo sobre a densidade do solo em dois sistemas de manejo do solo. R. Bras. Ci. Solo, 23:165-173, 1999.

DECAGON DEVICES. Disponível em: <http://www.decagon. com/assets/Uploads/AN-Determining-15-Bar-PermanentWilt-Water-Content-of-Soils-with-the-WP4C.pdf $>$. Acesso em: 10 de mar. de 2011.

DODD, I.C.; STIKIC, R. \& DAVIES, W.J. Chemical regulation of gas exchange and growth of plants in drying soil in the field. J. Exper. Bot., 47:1475-1490, 1996.

DIAS JUNIOR, M.S. \& MIRANDA, E.É.V. Comportamento da curva de compactação de cinco solos da região de Lavras (MG). Ci. Agrotec., 24:337-346, 2000. 
EHLERS, W.; KOPKE, U.; HESSE, F. \& BOHM, W. Penetration resistance and root growth of oats in the tilled an untilled loess soil. Soil Tillage Res., 3:261-275, 1983.

EMPRESA BRASILEIRA DE PESQUISA AGROPECUÁRIA EMBRAPA. Centro Nacional de Pesquisa de Solos. Manual de métodos de análise de solo. 2.ed. Rio de Janeiro, 1997. $212 \mathrm{p}$.

FABRIZZI, K.P.; GARCIA, F.O.; COSTA, J.L. \& PICONE, L.I. Soil water dynamics, physical properties and corn and wheat responses to minimum and no-tillage systems in the southern Pampas of Argentina. Soil Tillage Res., 81:57-69, 2005 .

FEDERAÇÃO BRASILEIRA DE PLANTIO DIRETO NA PALHA - FEBRAPDP. Disponível em: <http://www.febrapdp.org.

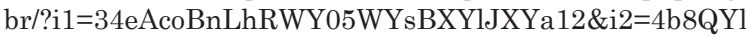
JXYfde\&i3=e46ARQBSZkBSYlJXwece\&i4=d4127e\&i5=3

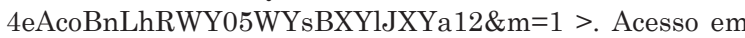
26 de mar. de 2011.

FERRERAS, L.A.; COSTA, J.L.; GARCIA, F.O. \& PECORARI. C. Effect of no-tillage on some soil physical properties of a structural degraded Petrocalcic Paleudoll of the southern "Pampa" of Argentina. Soil Tillage Res., 54:31-19, 2000.

FIGUEIREDO, L.H.A.; DIAS JUNIOR, M.S. \& FERREIRA, M.M. Umidade crítica de compactação máxima em resposta a sistemas de manejo num Latossolo Roxo. R. Bras. Ci. Solo, 24:487-493, 2000.

FIGUEIREDO, G.C. Avanços metodológicos e instrumentais em física do solo. Piracicaba, Escola Superior de Agricultura "Luiz de Queiroz", Universidade de São Paulo, 2010. 163p. (Tese de Doutorado)

FIGUEIREDO, G.C.; SILVA, A.P. \& TORMENA, C.A. Improvement of a testing apparatus for dynamometry: precedures for penetrometry and influence of strain rate to quantify the tensile strength of soil aggregates. R. Bras. Ci. Solo., 35:373-387, 2011.

GRABLE, A.R. \& SIEMER, E.G. Effects of bulk density, aggregate size, and soil water suction on oxygen diffusion, redox potential and elongation of corn roots. Soil Sci. Soc. Am. J., 32:180-186, 1968.

GRANTZ, S.A. \& SLINKER, B.K. Primer of applied regression and analysis of variance. New York, McGraw Hill, 1990. $777 \mathrm{p}$.

HAKANSSON, I. \& LIPIEC, J. A review of the usefulness of relative bulk density values in studies of soil structure and compactatiom. Soil Tillage Res., 53:71-85, 2000.

HAKANSSON, I. A method for characterizing the state of compactness of the plough layer. Soil Tillage Res., 16:105$120,1990$.

HOBBS, P.R. Conservation agriculture: What is it and why is it important for future sustainable food production? J. Agric. Sci., 145:127-137, 2007.

IMHOFF, S.; SILVA, A.P.; DIAS JR., M.S. \& TORMENA, C.A. Aplicações da curva de resistência no controle da qualidade física de um solo sob pastagem. Pesq. Agropec. Bras, $35: 1493-1500,2000$
IMHOFF, S.; SILVA, A.P.; DIAS JÚNIOR, M.S. \& TORMENA, C.A. Quantificação de pressões críticas para o crescimento de plantas. R. Bras. Ci. Solo, 25:11-18, 2001.

JIN, H.; HONGWEN, L.; RABI G. RASAILY, R. G.; QINGJIE, W.; GUOHUA, C.; YANBO, S.; XIAODONG, Q. \& LIJIN, L. Soil properties and crop yields after 11 years of no tillage farming in wheat-maize cropping system in North China Plain. Soil Tillage Res., 113:48-54, 2011.

KLEIN, V.A. Densidade relativa - um indicador da qualidade física de um Latossolo Vermelho. R. Ci. Agrovet., 5:26-32, 2006.

KLEIN, V.A. \& CAMARA, R.K. Rendimento da soja e intervalo hídrico ótimo em Latossolo Vermelho sob plantio direto escarificado. R. Bras. Ci. Solo, 31:221-227, 2007.

KLUTE, A. Water retention: Laboratory methods. In: KLUTE, A., ed. Methods of soil analysis: Physical and mineralogical methods. 2.ed. Madison, America Society of Agronomy, 1986. p.635-660.

KOOLEN, A.J. Mechanics of soil compaction. In: SOANE, B.D. \& van OUWERKERK, C., eds. Soil compaction in crop production. Amsterdam, Elsevier Science, 1994. p.23-44.

LEÃO, T.P.; SILVA, A.P.; MACEDO, M.C.M.; IMHOFF, S. \& EUCLIDES, V.P.B. Intervalo hídrico ótimo na avaliação de sistemas de pastejo contínuo e rotacionado. R. Bras. Ci. Solo, 28:415-423, 2004

LEÃO, T.P.; SILVA, A.P.; PERFECT, E. \& TORMENA, C.A. An algorithm for calculating the least limiting water range of soil using SAS. Agron. J., 97:1210-1215, 2005.

LETEY, J. Relationship between soil physical properties and crop production. Adv. Soil Sci., 1:277-294, 1985.

LIPIEC, J.; HAKANSSON, I.; TARKIEWICZ, S. \& KOSSOWSKI, J. Soil physical properties and growth of spring barley as related to the degree of compactness of two soils. Soil Tillage Res., 19:307-317, 1991.

MASLE, J. Growth and stomatal responses of wheat seedings to spatial and temporal variations in soil strength of bi-layered soils. J. Exper. Bot., 49:1245-1257, 1998.

NOGUEIRA, J.B. Mecânica dos solos. Ensaios de Laboratório. São Carlos, EESC-USP, 1998. 248p.

OLIVEIRA, V.S.; ROLIM, M.M.; VASCONCELOS, R.F.B.; COSTA, Y.D.J. \& PEDROSA, E.M.R. Compactação de um argissolo amarelo distrocoeso submetido a diferentes manejos. R. Bras. Eng. Agric. Amb., 14:914-920, 2010.

PAYTON, M.E.; MILLER, A.E. \& RAUN, W.R. Testing statistical hypotheses using standard error bars and confidence intervals. Comm. Soil Sci. Plant. Anal., 31:547-551, 2000.

PETEAN, L.P.; TORMENA, C.A. \& ALVES, S.J. Intervalo hídrico ótimo de um Latossolo Vermelho distroférrico sob plantio direto em sistema de integração lavoura-pecuária. R. Bras. Ci. Solo, 34:1515-1526, 2010.

REICHARDT, K. Capacidade de campo. R. Bras. Ci. Solo, 12:211-16, 1988 
SAS Institute. SAS/STAT procedure guide for personal computer. 5.ed. Cary, 1999.

SAVAGE, M.J.; RITCHIE, J.T.; BLAND, W.L. \& DUGAS, W.A. Lower limit of soil water availability. Agron. J., 88:844851, 1996.

SILVA, A.P.; KAY, B.D. \& PERFECT, E. Characterization of the least limiting water range. Soil Sci. Soc. Am. J., 58:17751781, 1994.

SILVA, A.P.; TORMENA, C.A.; IMHOFF, S.C.; FIDALSKI, J. \& NEVES JÚNIOR, A. Intervalo hídrico ótimo e sua importância para as plantas. In: RIBEIRO, M.R.; NASCIMENTO, C.W.; RIBEIRO FILHO, M.R. \& CANTALICE, J.R.B., eds. Tópicos em ciência do solo. Viçosa, MG, Sociedade Brasileira de Ciência do Solo, 2009. v.6. p.1-30.

SO, H.B.; GRABSKI, A. \& DESBOROUGH, P. The impact of 14 years of conventional and no-till culivation on the physical properties and crop yields of a loam soil at Grafton NSW, Australia. Soil Tillage Res., 104:180-184, 2009.

STRUDLEY, M.W.; GREEN, T.R. \& ASCOUGH II, J.C. Tillage effects on soil hydraulic properties in space and time: State of the science. Soil Tillage Res., 99:4-48, 2008.

SUZUKI, L.E.A. S.; REICHERT, J.M.; REINERT, D.J. \& LIMA, C.L.R. Grau de compactação, propriedades físicas e rendimento de culturas em Latossolo e Argissolo. Pesq. Agropec. Bras., 42:1159-1167, 2007.

TAYLOR, H.M.; ROBERSON, G.M. \& PARKER JR, J.J. Soil strength-root penetration relations to medium to coarsetextured soil materials. Soil Sci., 102:18-22, 1966.
TORMENA, C.A.; ARAÚJO, M.A.; FIDALSKI, J. \& COSTA, J.M. Variação temporal do intervalo hídrico ótimo de um Latossolo Vermelho distroférrico em sistemas de plantio direto. R. Bras. Ci. Solo, 31:211-219, 2007.

TORMENA, C.A.; SILVA, A.P. \& LIBARDI, P.L. Caracterização do intervalo hídrico ótimo de um Latossolo Roxo sob plantio direto. R. Bras. Ci. Solo, 22:573-581, 1998.

TORMENA, C.A.; SILVA, A.P. \& LIBARDI, P.L. Soil physical quality of a Brazilian Oxisol under two tillage systems using the least limiting water range approach. Soil Tillage Res., 52:223-232, 1999.

TORRES, E. \& SARAIVA, O.F. Camadas de impedimento mecânico do solo em sistemas agrícolas com a soja. Londrina, Embrapa Soja, 1999. 58p. (Embrapa soja. Circular Técnica, 23)

VEIGA, M.; HORN, R.; REINERT, D.J. \& REICHERT, J.M. Soil compressibility and penetrability of an Oxisol from southern Brazil, as affected by long-term tillage systems. Soil Tillage Res., 92:104-113, 2007.

VEPRASKAS, M.J. Cone index of loamy sands as influenced by pore size distribution and effective stress. Soil Sci. Soc. Am. J., 48:1220-1225, 1984.

VIEIRA, M.L. \& KLEIN, V.A. Propriedades físico-hídricas de um Latossolo Vermelho submetido a diferentes sistemas de manejo. R. Bras. Ci. Solo, 31:1271-1280, 2007.

WEIRICH NETO, P.H.; ROSA, A.L.T. \& GOMES, J.A. Suscetibilidade de dois tipos de solo à compactação. R. Bras. Eng. Agric. Amb., 6:349-353, 2002. 